\title{
Regulation of Pax7 protein levels by caspase-3 and proteasome activity in differentiating myoblasts
}

\author{
Hugo C. Olguín ${ }^{1 *}$ \\ ' Departamento de Biología Celular y Molecular, Facultad de Ciencias Biológicas, Pontificia Universidad Católica de Chile. Alameda 340, Santiago. Chile.
}

\begin{abstract}
The transcription factor Pax7 negatively regulates the activity of the muscle regulatory transcription factor MyoD, preventing muscle precursor cells from undergoing terminal differentiation. In this context, the ratio between Pax7 and MyoD protein levels is thought to be critical in allowing myogenesis to proceed or to maintain the undifferentiated muscle precursor state. We have previously shown that Pax7 is subject to rapid down regulation in differentiating myoblasts, via a proteasome-dependent pathway. Here we present evidence indicating that Pax7 is also subject to caspase-3-dependent regulation. Furthermore, simultaneous inhibition of caspase-3 and proteasome activity induced further accumulation of Pax7 protein in differentiating myoblasts. These results suggest that at early stages of muscle differentiation, Pax7 levels are regulated by at least two independent mechanisms involving caspase-3 and proteasome activity.
\end{abstract}

Key words: Pax7, caspase-3, calpains, proteasome, satellite cells, muscle differentiation.

\section{INTRODUCTION}

Skeletal muscle regeneration depends on resident stem cells maintained in a non-proliferative state, known as satellite cells [Mauro 1961; Schultz Gibson and Champion 1978]. The transcription factor Pax7 is expressed in quiescent satellite cells and plays critical roles in satellite cell biogenesis, survival and renewal [Lagha et al. 2008; Rudnicki et al. 2008]. Upon muscle injury, satellite cells become activated, proliferate and induce the expression of the muscle regulatory transcription factors (MRFs) MyoD and Myf-5 [Hawke and Garry 2001; Chargé and Rudnicki 2004]. Adult myoblasts commit to terminal differentiation by inducing the expression of the MRF myogenin, down-regulate Pax7 expression and ultimately fuse to one another forming multinucleated muscle fibers [Ciciliot and Schiaffino 2010]. Pax7 can repress myogenesis by regulating MyoD activity [Olguin and Olwin 2004; Olguín et al. 2007; Kumar et al. 2009]. Thus, the ratio between Pax7 and MyoD protein levels appears to be critical in allowing terminal differentiation (low Pax7/high MyoD) or maintaining an undifferentiated progenitor state (high Pax7/ low MyoD) [Olguín et al. 2007], both critical for proper muscle regeneration [Brack et al. 2008].

We have previously shown that down regulation of Pax7 in differentiating myoblasts is partially prevented by proteasome inhibition [Olguín et al. 2007]. Here we provide evidence for a second pathway regulating Pax7 expression and show that inhibition of caspase-3 results in increased Pax7 levels in differentiating MM14 myoblasts. Moreover, an additive effect was observed when caspase- 3 and proteasome where simultaneously inhibited, suggesting that these pathways regulate Pax7 protein levels independently.

\section{MATERIALS AND METHODS}

Cell culture and transfections

MM14 cells were cultured in F12-C, 15\% horse serum, and 500 pM FGF-2 at $37^{\circ} \mathrm{C}$ and $5 \% \mathrm{CO}_{2}$. Differentiation was induced by culturing myoblasts in F12-C and $10 \%$ horse serum. For myctagged ubiquitin expression, MM14 myoblasts were transfected with pCMV-myc-ubiquitin vector (a gift by Dr. Kristen Barthel, MCD Biology, University of Colorado at Boulder) as described previously [Olguin et al. 2007].

Immunoprecipitation and Western Blot

Myc-tagged-ubiquitin transfected cells were cultured for $24 \mathrm{~h}$ after transfection or switched to differentiation medium $18 \mathrm{~h}$ after transfection and cultured for additional $24 \mathrm{~h}$. Cells were incubated with $25 \mu \mathrm{M}$ proteasome inhibitor MG132 (EMDCalbiochem) for 6-8 $\mathrm{h}$ before lysis. Immunoprecipitation of ubiquitinated proteins was performed using a mouse monoclonal anti-myc antibody (clone 9B11, Cell Signaling. 1:1000 dilution). Total protein concentrations were normalized to $0.5-1 \mathrm{mg} / \mathrm{ml}$ and equal amount proteins were subject to immunoprecipitation as described [Olguín et al. 2007]. When indicated, cells were incubated with $20 \mu \mathrm{M}$ of Z-FA-FMK (inactive caspase inhibitor), $10-20 \mu \mathrm{M}$ Z-VAD-FMK (general caspase inhibitor), $20 \mu \mathrm{M}$ of Z-DEVD-FMK (caspase-3 inhibitor; BD-Pharmingen) or $2.5-5 \mu \mathrm{M}$ calpastatin-peptide (calpain $1 / 2$ inhibitor, EMD-Calbiochem) for $6 \mathrm{~h}$ prior to lysis. For proteasome and calpain/caspase inhibition, cells were coincubated with $25 \mu \mathrm{M}$ MG132 or vehicle (DMSO) and the specified calpain/caspase inhibitors $6 \mathrm{~h}$ prior lysis. Mouse

\footnotetext{
* Corresponding Author: Hugo C. Olguín. Laboratorio de Reparación Tisular y Células Troncales Adultas, Departamento de Biología Celular y Molecular, Facultad de Ciencias Biológicas, Pontificia Universidad Católica de Chile, Alameda 340, Santiago, Chile. 56-02-654 1860 (office) - 56-02-354 2660 (fax) e-mail: holguin@bio.puc.cl
} 
monoclonal anti-Pax7 antibody (Developmental Studies Hybridoma Bank, cell culture supernatant. 1:10 dilution) was used for Pax7 immunoprecipitation and Western blot detection. Anti-mouse HRP-conjugated secondary antibodies (Promega) were used at 1:5,000 and HRP activity was visualized using the ECL Plus Western Blotting Detection System (GE Healthcare). X-ray films were scanned (Powerlook 1120 scanner; UMAX), and analyzed (ImageJ; NIH) for figure preparation.

\section{RESULTS AND DISCUSSION}

It is currently unknown if $\operatorname{Pax} 7$ is a direct proteasome target or if proteasome-dependent degradation of Pax7 co-factors results in $\operatorname{Pax} 7$ down regulation by alternative pathways. Protein poly-ubiquitination is required for proteasomedependent degradation of many cellular proteins [Finley 2009; Stadtmueller and Hill 2011]. Therefore, we reasoned that Pax7 (ubiquitin-modified and/or associated with ubiquitinated proteins) could be selectively enriched after isolation of ubiquitinated proteins following proteasome inhibition in differentiating myoblasts. Therefore, we performed immunoprecipitation of total ubiquitinated proteins from MM14 myoblasts expressing myc-tagged ubiquitin, followed by western blot analysis for Pax7. Unexpectedly, two distinct protein-band species were consistently detected in the immunoprecipitated fractions: i) low molecular weight bands ( 40-30 kDa; Fig. 1A), and ii) a protein band corresponding to the predicted full-length Pax7 (not shown; Bustos FJ., manuscript in preparation). When the anti-myc epitope antibody was omitted from the immunoprecipitation step, neither protein species were detected (Fig. 1A), suggesting that low-molecular weight immunoprecipitated proteins are bona fide Pax7 derived proteins/fragments. Alternativespliced Pax7 transcripts have been previously described, which are thought to impose minor changes in the fulllength Pax7 protein sequence. A truncated mRNA has also been described, coding for a putative N-terminus fragment of the Pax7 [Ziman and Kay 1997; Ziman and Kay 1998]. However, the Pax7 monoclonal antibody used in this study
A.
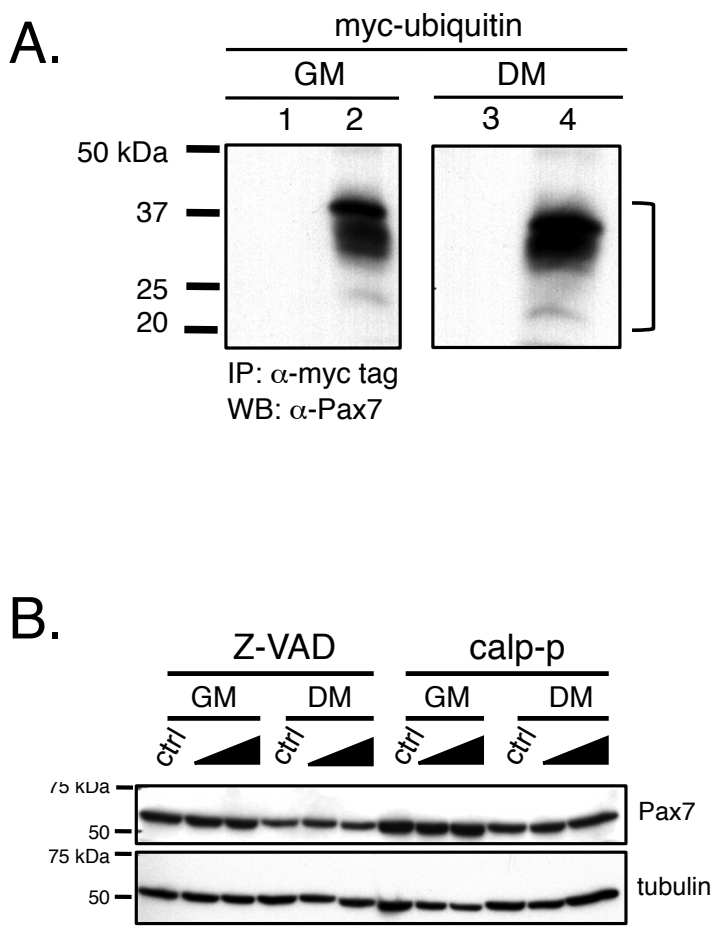

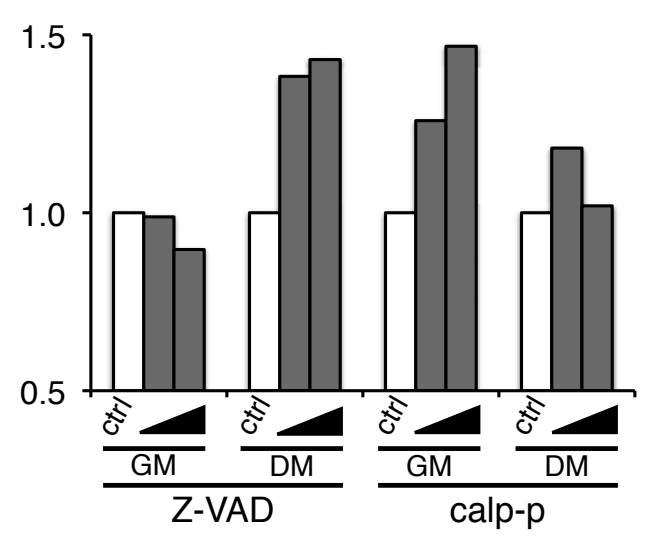

Figure 1: Pax7 protein regulation by proteolytic cleavage. A. MM14 myoblasts were transfected with pCMV-myc-tag ubiquitin and maintained in growth medium (GM) or differentiation medium (DM) for $24 \mathrm{~h}$ prior to treatment with proteosome inhibitor MG132 and lysis. Western blot analysis after immunoprecipitation of the myc-epitope (lanes 2,4), revealed a series of low molecular weight bands reactive to the Pax7 antibody (bracket). As control, anti-myc antibody was omitted from immunoprecipitation step (lanes 1,3). Representative of two independent experiments. B. MM14 myoblast induced to differentiate for $18 \mathrm{~h}$ (DM) or maintained in growth medium (GM) were treated with general caspase inhibitor (Z-VAD-FMK) or calpain $1 / 2$ inhibitor (calpastatin peptide) prior to lysis and immunoprecipitation of Pax7 followed by Western blot (left panel). Densitometry analysis revealed accumulation of Pax7 upon caspase inhibition in differentiating myoblasts (black bars). Note that inhibition of calpain activity resulted in Pax7 accumulation only in proliferating myoblasts (gray bars). Representative of two independent experiments. 
recognizes an epitope at the C-terminus region [Kawakami et al. 1997], suggesting that low-molecular weight bands (Fig. 1A) represent Pax7 C-terminus fragments derived from regulated proteolytic cleavage. Accordingly, the intensity of the low molecular weight bands increased when the myoblasts were cultured in differentiating conditions (Fig.1A), which correlates with previously described increase in Pax7 degradation upon induction of muscle differentiation [Olguin et al. 2007].

Members of the calpains and caspases families of proteases play critical roles in muscle differentiation [Fernando et al. 2002; Liang et al. 2006; Larsen et al. 2010]. Based on the observations described above and in silico analysis of Pax7 sequence indicating potential calpain and caspases cleavage sites (Olguín H., unpublished results), we hypothesized that calpain and/or caspases dependent pathways could be involved in the post-translational regulation of Pax7. Thus, we evaluated the effect of calpain and caspase activity inhibition on full length-Pax7 protein levels in MM14 myoblasts cultured in the presence or absence of a calpain $1 / 2$ inhibitor (calpastatin-peptide) or a general caspase inhibitor (Z-VADFMK). Interestingly, increased Pax7 levels were observed upon calpain inhibition in proliferating myoblasts (Fig. 1B). No calpain-dependent effect was observed when myoblasts were cultured under differentiating conditions (Fig. 1B). To our knowledge this is the first evidence indicating that Pax7 protein levels are subject to post-translational control in proliferating myoblasts, and its significance needs to be elucidated. On the other hand, Pax7 levels were up-regulated in the presence of the caspase inhibitor only in cells cultured in differentiating conditions. Among different caspases isoforms, caspase- 3 has been specifically involved in the initiation of muscle differentiation and myoblast fusion [Fernando et al. 2002; Larsen et al. 2010]. Accordingly, we observed a $2.5-$ fold increase in Pax7 protein levels upon specific caspase-3 inhibition (Fig. 2). This effect resembles the up-regulation of Pax7 in myoblasts treated with the general caspase inhibitor, suggesting that caspase-3 is the main caspase isoform responsible for Pax7 regulation in differentiating myoblasts. Inactive caspase inhibitor has no detectable effect on Pax7 protein (Fig. 2).

Many contractile muscle proteins are modified by proteolytic enzymes prior to complete breakdown via proteasome degradation [Attaix et al. 2005]. Consequently, we asked whether caspase-3 activity was required for the previously described proteasome-dependent Pax7 down regulation [Olguín et al. 2007]. If acting upstream of the proteasome, we hypothesized that inhibition of caspase- 3 would prevent further increase of Pax7 levels upon proteaseome inactivation. Unexpectedly, proteasome inhibition induced up regulation of Pax7 protein in cells treated with either general or caspase-3 inhibitors (Fig. 3A). Densitometry analysis indicated that Pax7 levels were increased $>2$-fold upon proteasome inhibition in both untreated and Z-DEVDFMK treated cells (Fig. 3B). As shown previously, calpaininhibition in differentiating myoblasts had no detectable effect on Pax7 levels and did not affect accumulation of Pax7 upon proteasome inhibition (Fig. 3B). Together, these results indicate that caspase- 3 and the proteasome participate in separate pathways to down regulate Pax7 protein upon initiation of muscle differentiation.
Pax7 and myogenin expression are mutually exclusive [Olguin and Olwin, 2004; Zammit et al. 2004; Olguín et al. 2007]. On the other hand, caspase- 3 inhibition impairs up regulation of myogenin [Fernando et al. 2002]. In light of the results presented here, it is tempting to propose a feedback loop between myogenin induction and caspase-3 activation to down regulate $\mathrm{Pax} 7$, reinforcing the non-reversible commitment to terminal differentiation. Current efforts are directed toward understanding the specific roles of calpain/ caspase and proteasome-dependent pathways and the underlying molecular regulation that controls Pax7 function during muscle development and regeneration.
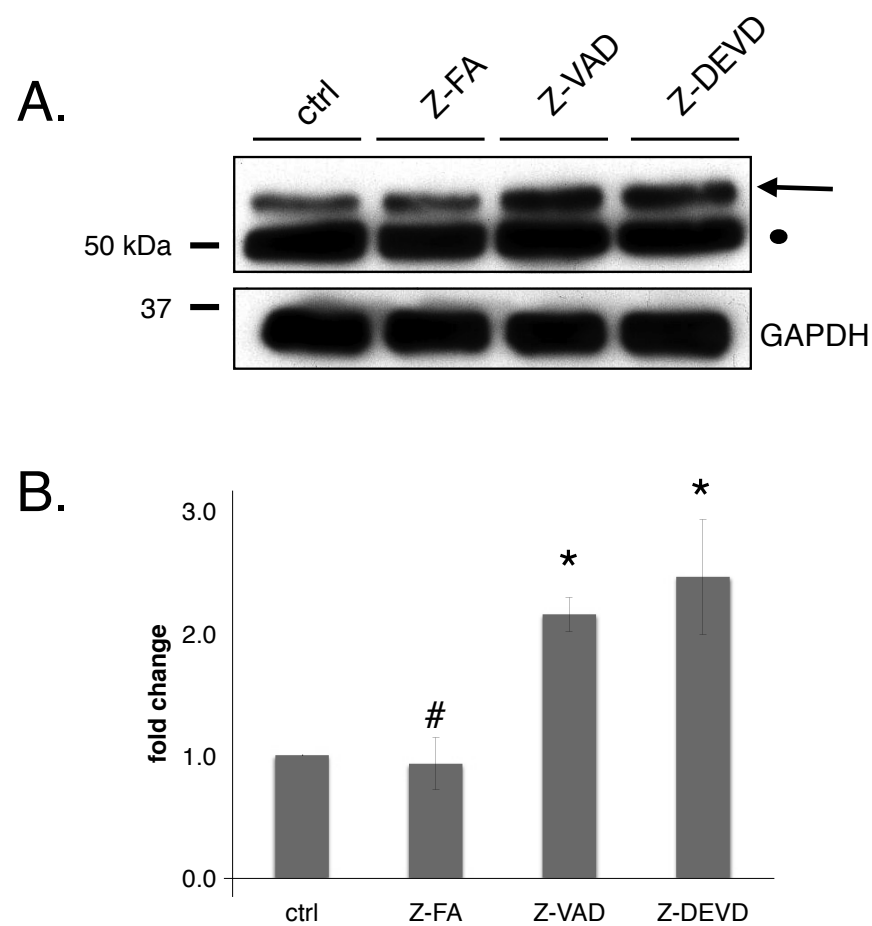

Figure 2: Down regulation of Pax7 levels is prevented by specific inhibition of caspase-3 activity. MM14 myoblasts cultured in differentiating conditions for $18 \mathrm{~h}$ were treated with inactive caspase inhibitor (Z-FA), general caspase inhibitor (Z-VAD) or caspase-3 inhibitor (Z-DEVD), prior to lysis and immunoprecipitation for Pax7. Upper panel: representative Western blot for Pax7 and GAPDH. Lower panel: densitometry quantification revealed that capase- 3 inhibition results in equivalent accumulation of Pax7 over control (arrow; $2.45 \pm 0.47$ fold change) when compare to Z-VAD ( $2.15 \pm 0.13$ fold change). Black circle $=$ IgG heavy chain. GAPDH=loading control from immunoprecipitation inputs. Mean values from 3 independent experiments. Error bars $=$ standard deviation. ${ }^{*} p<0.01$; \#: not statistically different from control. 

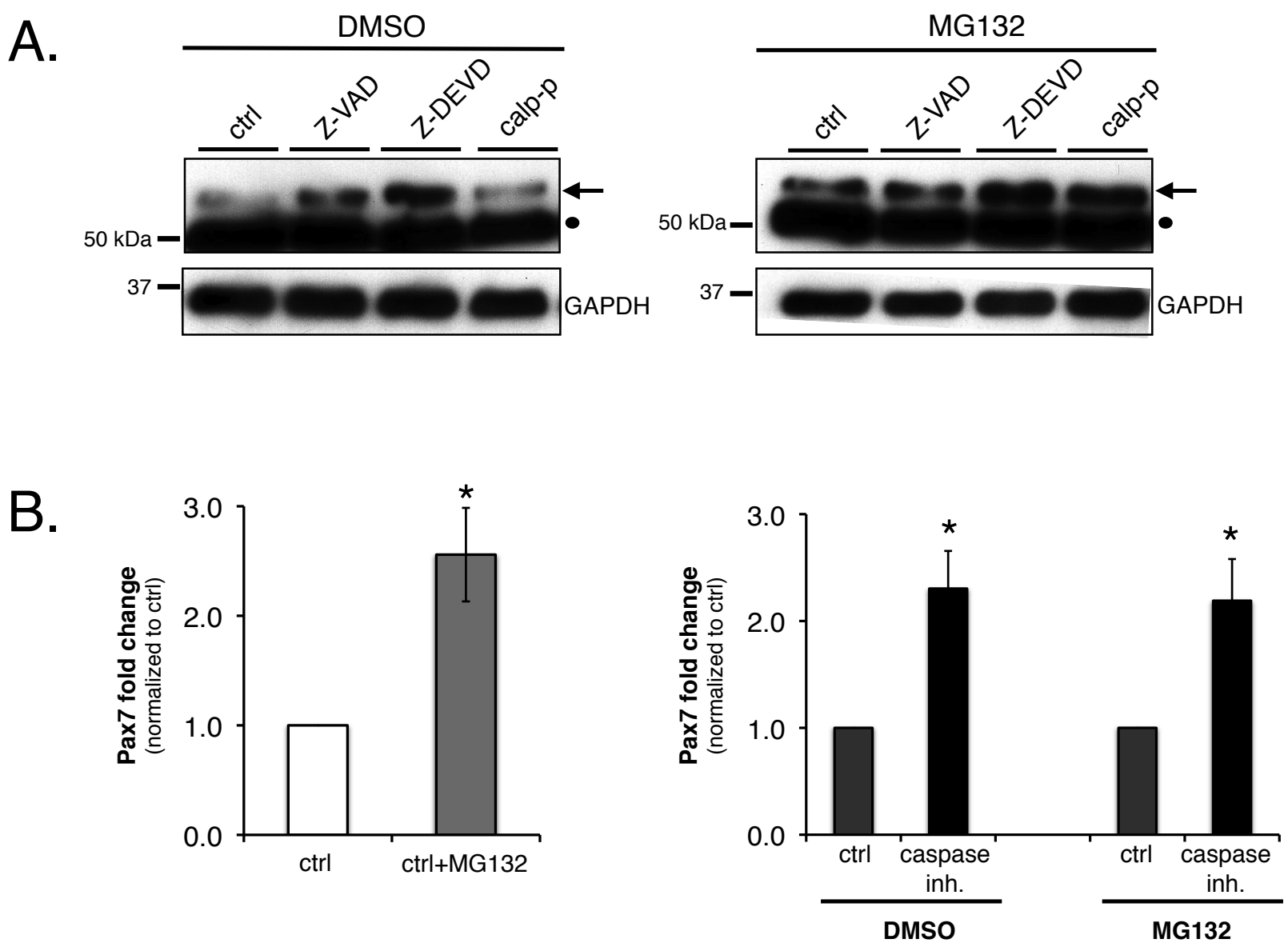

Figure 3: Caspase-3-dependent and proteasome-dependent pathways regulate Pax7 independently. A. MM14 myoblasts where differentiated for $18 \mathrm{~h}$ in the presence or absence of Z-VAD; Z-DEVD or calpain inhibitor (calp-p). $6 \mathrm{~h}$ prior to lysis, cells were treated with proteasome inhibitor MG132 or vehicle (DMSO). Extracts were analyzed as in Figure 2. Pax7 (arrow) accumulates upon MG132 treatment in control and calpastain-p co-treatments (left panel). Black circle= IgG heavy chain. B. Comparative densitometry analysis from at least two independent analyses reveals a $2.5 \pm 0.42$ fold increase in the accumulation of Pax7 upon proteasome inhibition (left panel). Despite this increase, when compared to their respective control values, the $>2$ fold change in Pax 7 levels upon caspase inhibition ( $2.3 \pm 0.35$ fold change) is maintained upon simultaneous caspase and proteasome inhibition ( $2.19 \pm 0.19$ fold change), suggesting two parallel pathways. Mean values from two (caspase inhibition) and three (control plus or minus MG132) independent experiments. Error bars= standard deviation. ${ }^{*} \mathrm{p}<0.01$.

\section{ACKNOWLEDGMENTS}

This work was supported by Internal Grant from P. Catholic University of Chile (VRAID \#20/2009 and \#17/2010) to HO.

\section{REFERENCES}

ATTAIX D, VENTADOUR S, CODRAN A, BÉCHET D, TAILLANDIER $\mathrm{D}$, and COMBARET L (2005). The ubiquitin-proteasome system and skeletal muscle wasting. Essays Biochem 41:173-186.

BRACK AS, CONBOY IM, CONBOY MJ, SHEN J, and RANDO TA (2008). A temporal switch from notch to Wnt signaling in muscle stem cells is necessary for normal adult myogenesis. Cell Stem Cell 2:50-59.

CHARGÉ SB, and RUDNICKI MA (2004). Cellular and molecular regulation of muscle regeneration. Physiol Rev 84:209-238.

CICILIOT S, and SCHIAFFINO S (2010). Regeneration of mammalian skeletal muscle. Basic mechanisms and clinical implications. Curr Pharm Des 16:906-914.

FERNANDO P, KELLY JF, BALAZSI K, SLACK RS, and MEGENEY LA (2002). Caspase 3 activity is required for skeletal muscle differentiation. Proc Natl Acad Sci USA 99:11025-11030.
FINLEY D (2009). Recognition and processing of ubiquitin-protein conjugates by the proteasome. Annu Rev Biochem 78:477-513.

HAWKE TJ, and GARRY DJ (2001). Myogenic satellite cells: physiology to molecular biology. J Appl Physiol 91:534-551.

KAWAKAMI A, KIMURA-KAWAKAMI M, NOMURA T, and FUJISAWA H (1997). Distributions of PAX6 and PAX7 proteins suggest their involvement in both early and late phases of chick brain development. Mech Dev 66:119-130.

KUMAR D, SHADRACH JL, WAGERS AJ, and LASSAR AB (2009). Id3 is a direct transcriptional target of Pax7 in quiescent satellite cells. Mol Biol Cell 20:3170-3177.

LAGHA M, SATO T, BAJARD L, DAUBAS P, ESNER M, MONTARRAS D, RELAIX F, and BUCKINGHAM M (2008). Regulation of skeletal muscle stem cell behavior by Pax3 and Pax7. Cold Spring Harb Symp Quant Biol 73:307-315.

LARSEN BD, RAMPALLI S, BURNS LE, BRUNETTE S, DILWORTH FJ, and MEGENEY LA (2010). Caspase 3/ caspase-activated DNase promote cell differentiation by inducing DNA strand breaks. Proc Natl Acad Sci U S A 107:4230-4235.

LIANG YC, YEH JY, FORSBERG NE, and OU BR (2006). Involvement of $\mathrm{mu}-$ and $\mathrm{m}$-calpains and protein kinase $\mathrm{C}$ isoforms in L8 myoblast differentiation. Int J Biochem Cell Biol 38:662-670. 
MAURO A (1961). Satellite cell of skeletal muscle fibers. J Biophys Biochem Cytol 9:493-495.

OLGUÍN HC, and OLWIN BB (2004). Pax-7 up-regulation inhibits myogenesis and cell cycle progression in satellite cells: a potential mechanism for self-renewal. Dev Biol 275:375-388.

OLGUÍN HC, YANG Z, TAPSCOTT SJ, and OLWIN BB (2007). Reciprocal inhibition between Pax7 and muscle regulatory factors modulates myogenic cell fate determination. J Cell Biol 177:769-779.

RUDNICKI MA, LE GRAND F, MCKINNELL I, and KUANG S (2008). The molecular regulation of muscle stem cell function. Cold Spring Harb Symp Quant Biol 73:323-331.

SCHULTZ E, and MCCORMICK KM (1994). Skeletal muscle satellite cells. Rev Physiol Biochem Pharmacol 123:213-257.
SCHULTZ E, GIBSON MC, and CHAMPION T (1978). Satellite cells are mitotically quiescent in mature mouse muscle: an EM and radioautographic study. J Exp Zool 206:451-456.

STADTMUELLER BM, and HILL CP (2011). Proteasome activators. Mol Cell 41:8-19.

ZAMMIT PS, GOLDING JP, NAGATA Y, HUDON V, PARTRIDGE TA, and BEAUCHAMP JR (2004). Muscle satellite cells adopt divergent fates: a mechanism for self-renewal? J Cell Biol 166:347-357.

ZIMAN MR, and KAY PH (1998). Differential expression of four alternate Pax7 paired box transcripts is influenced by organ- and strain-specific factors in adult mice. Gene 217:77-81.

ZIMAN MR, FLETCHER S, and KAY PH (1997). Alternate Pax7 transcripts are expressed specifically in skeletal muscle, brain and other organs of adult mice. Int J Biochem Cell Biol 29:1029-1036. 
